\title{
Oversampling and Undersampling in de Branges Spaces Arising from Regular Schrödinger Operators
}

\author{
Luis O. Silva ${ }^{1}$ (D) Julio H. Toloza ${ }^{2}$ (D) Alfredo Uribe ${ }^{1}$ \\ Received: 2 May 2018 / Accepted: 1 October 2018 / Published online: 12 October 2018 \\ (c) The Author(s) 2018
}

\begin{abstract}
The classical results on oversampling and undersampling (or aliasing) of functions in Paley-Wiener spaces are generalized to the case of functions in de Branges spaces arising from regular Schrödinger operators with a wide range of potentials.
\end{abstract}

Keywords de Branges spaces · Schrödinger operators · Sampling theory

Mathematics Subject Classification 47B32 $\cdot 41 \mathrm{~A} 05 \cdot 34 \mathrm{~L} 40$

\section{Introduction}

This paper deals with the subject of oversampling and undersampling - the latter also known as aliasing in the engineering and signal processing literature-in the context of de Branges Hilbert spaces of entire functions ( $\mathrm{dB}$ spaces for short). These notions play a prominent role in the theory of Paley-Wiener spaces [15,23]. Since Paley-

Communicated by Harry Dym.

Luis O. Silva: Supported by UNAM-DGAPA-PAPIIT IN110818 and SEP-CONACYT CB-2015 254062. Julio H. Toloza: Partially supported by CONICET (Argentina) through Grant PIP 11220150100327 CO.

$凶 \quad$ Luis O. Silva

silva@iimas.unam.mx

Julio H. Toloza

julio.toloza@uns.edu.ar

Alfredo Uribe

alfredo.uribe.83@ciencias.unam.mx

1 Departamento de Física Matemática, Instituto de Investigaciones en Matemáticas Aplicadas y en Sistemas, Universidad Nacional Autónoma de México, C.P. 04510 Mexico City, Mexico

2 INMABB, Departamento de Matemática, Universidad Nacional del Sur (UNS)-CONICET, Bahía Blanca, Argentina 
Wiener spaces are leading examples of $\mathrm{dB}$ spaces, questions related to oversampling and undersampling in $\mathrm{dB}$ spaces emerge naturally.

Paley-Wiener spaces stem from the Fourier transform of functions with given compact support centred at zero, viz.,

$$
\mathcal{P} \mathcal{W}_{a}:=\left\{f(z)=\int_{-a}^{a} e^{-i x z} \phi(x) d x: \phi \in L_{2}(-a, a)\right\} .
$$

By the Whittaker-Shannon-Kotel'nikov theorem, any function $f(z) \in \mathcal{P} \mathcal{W}_{a}$ is decomposed as follows.

$$
f(z)=\sum_{n \in \mathbb{Z}} f\left(\frac{n \pi}{a}\right) \mathcal{G}_{a}\left(z, \frac{n \pi}{a}\right), \quad \mathcal{G}_{a}(z, t):=\frac{\sin [a(z-t)]}{a(z-t)}
$$

where the convergence of the series is uniform in any compact subset of $\mathbb{C}$. The function $\mathcal{G}_{a}(z, t)$ is referred to as the sampling kernel.

In oversampling, the starting point is a function $f(z) \in \mathcal{P} \mathcal{W}_{a} \subset \mathcal{P} \mathcal{W}_{b}(a<b)$. Then, in addition to (1.1), one has

$$
f(z)=\sum_{n \in \mathbb{Z}} f\left(\frac{n \pi}{b}\right) \mathcal{G}_{b}\left(z, \frac{n \pi}{b}\right)
$$

Moreover, $f(z)$ admits a different representation

$$
f(z)=\sum_{n \in \mathbb{Z}} f\left(\frac{n \pi}{b}\right) \widetilde{\mathcal{G}}_{a b}\left(z, \frac{n \pi}{b}\right)
$$

with a modified sampling kernel $\widetilde{\mathcal{G}}_{a b}(z, t)$ depending on $a$ and $b$ (see [15, Thm. 7.2.5]). While the convergence of the sampling formula (1.1) is unaffected by $l_{2}$ perturbations of the samples $f\left(\frac{n \pi}{a}\right)$, formula (1.2) is more robust because it is convergent even under $l_{\infty}$ perturbations of the samples. That is, if the sequence $\left\{\epsilon_{n}\right\}_{n \in \mathbb{Z}}$ is bounded and one defines

$$
\widetilde{f}(z):=\sum_{n \in \mathbb{Z}}\left[f\left(\frac{n \pi}{b}\right)+\epsilon_{n}\right] \widetilde{\mathcal{G}}_{a b}\left(z, \frac{n \pi}{b}\right),
$$

then $|f(z)-\tilde{f}(z)|$ is uniformly bounded in compact subsets of $\mathbb{C}$ [15, Thm. 7.2.5].

Undersampling, on the other hand, looks for the approximation of a function $f(z)$ in $\mathcal{P} \mathcal{W}_{b} \backslash \mathcal{P} \mathcal{W}_{a}$ by another one formally constructed using the sampling formula (1.1), namely,

$$
\widehat{f}(z)=\sum_{n \in \mathbb{Z}} f\left(\frac{n \pi}{a}\right) \mathcal{G}_{a}\left(z, \frac{n \pi}{a}\right) .
$$

The series in (1.4) is indeed convergent and, moreover, $|f(z)-\widehat{f}(z)|$ is uniformly bounded in compact subsets of $\mathbb{C}$. Formula (1.4) yields in fact an approximation not only for functions in $\mathcal{P} \mathcal{W}_{b} \backslash \mathcal{P} \mathcal{W}_{a}$ but for the Fourier transform of elements in $L_{1}(\mathbb{R}) \cap L_{2}(\mathbb{R})[15$, Thm. 7.2.9]. 
Oversampling and undersampling are, to some extent, consequences of the fact that the chain of Paley-Wiener spaces $\mathcal{P} \mathcal{W}_{s}, s \in(0, \infty)$, is totally ordered by inclusion. As this is a property shared by all dB spaces in the precise sense of [4, Thm.35], it is expected that analogous notions should make sense in this latter class of spaces. We note that sampling formulas generalizing (1.1) are known for arbitrary reproducing kernel Hilbert spaces (see e.g. Kramer-type formulas in [7,8,18,20]), dB spaces among them. Analysis of error due to noisy samples and aliasing, among other sources, in Paley-Wiener spaces goes back at least to [14]. More recent literature on the subject is, for instance, [1-3,12]. However, to the best of our knowledge, estimates for oversampling and undersampling are not known for $\mathrm{dB}$ spaces apart from the Paley-Wiener class.

A function $f(z)$ belonging to a $\mathrm{dB}$ space $\mathcal{B}$ obviously admits a representation in terms of an orthogonal basis. In particular,

$$
f(z)=\sum_{t \in \operatorname{spec}(S(\gamma))} f(t) \frac{k(z, t)}{k(t, t)}
$$

where $k(z, w)$ is the reproducing kernel of $\mathcal{B}$ and $S(\gamma)$ is a canonical selfadjoint extension of the operator of multiplication by the independent variable in $\mathcal{B}$. The expansion (1.5) is a sampling formula with $k(z, t) / k(t, t)$ being its sampling kernel. Note that (1.1) is a particular realization of (1.5) for the $\mathrm{dB}$ space $\mathcal{P} \mathcal{W}_{a}$.

In order to obtain oversampling and undersampling estimates in analogy to the Paley-Wiener case, we look into $\mathrm{dB}$ spaces of the form

$$
\mathcal{B}_{s}=\left\{f(z)=\int_{0}^{s} \xi(x, z) \phi(x) d x: \phi \in L_{2}(0, s)\right\},
$$

where $\xi(x, z)$ solves

$$
-\frac{d^{2}}{d x^{2}} \varphi+V(x) \varphi=z \varphi, \quad x \in(0, s), \quad z \in \mathbb{C},
$$

for some $s \in(0, \infty)$ and with Neumann boundary condition at $x=0$ (see Sect. 2). Here $V \in L_{1}(0, s)$ is a real function. By construction $\mathcal{B}_{s} \subset \mathcal{B}_{s^{\prime}}$ whenever $s<s^{\prime}$ (for more on this, see [17]).

Define

$$
\mathcal{K}_{s}(z, t):=\frac{k_{s}(z, t)}{k_{s}(t, t)},
$$

where $k_{S}(z, w)$ is the reproducing kernel of the space $\mathcal{B}_{s}$. If $S_{S}(\gamma)$ is a selfadjoint extension of the multiplication operator in $\mathcal{B}_{s}$, then any $f(z) \in \mathcal{B}_{s}$ has the representation

$$
f(z)=\sum_{t \in \operatorname{spec}\left(S_{s}(\gamma)\right)} f(t) \mathcal{K}_{s}(z, t) .
$$

Our main results are Theorems 3.6 and 4.7, which can be summarized as follows: 
Theorem (oversampling) Assume that $V$ is real-valued and in $A C[0, \pi]$ (the set of absolutely continuous functions in $[0, \pi])$. Consider an arbitrary $f(z) \in \mathcal{B}_{a}$, where $a \in(0, \pi)$. For a given $\left\{\epsilon_{t}\right\} \in l_{\infty}$, define

$$
\tilde{f}(z):=\sum_{t \in \operatorname{spec}\left(S_{\pi}(\pi / 2)\right)} \tilde{\mathcal{K}}_{a \pi}(z, t)\left(f(t)+\epsilon_{t}\right),
$$

where $\widetilde{\mathcal{K}}_{a b}(z, t)$ is given in (3.6). Then, for every compact set $K$ of $\mathbb{C}$, there is a constant $C(a, K, V)>0$ such that

$$
|f(z)-\tilde{f}(z)| \leq C(a, K, V)\|\epsilon\|_{\infty}, \quad z \in K
$$

We remark that the bound is uniform for $f(z) \in \mathcal{B}_{a}$. Note that $\widetilde{\mathcal{K}}_{a b}(z, t)$ is a modified sampling kernel analogous to the one in (1.3).

Theorem (undersampling) Assume $V$ is real-valued and in $A C[0, b]$ with $b>\pi$. Given $g(z) \in \mathcal{B}_{b} \backslash \mathcal{B}_{\pi}$, define

$$
\widehat{g}(z):=\sum_{t \in \operatorname{spec}\left(S_{\pi}(\pi / 2)\right)} g(t) \mathcal{K}_{\pi}(z, t) .
$$

Then, for each compact set $K \subset \mathbb{C}$, there is a constant $D(b, K, V)>0$ such that

$$
|g(z)-\widehat{g}(z)| \leq D(b, K, V) \int_{\pi}^{b}|\psi(x)| d x
$$

uniformly on $K$, where $\psi \in L_{2}(0, b)$ obeys $g(z)=\langle\xi(\cdot, \bar{z}), \psi(\cdot)\rangle_{L_{2}(0, b)}$.

These results are somewhat limited in several respects. First, we show oversampling relative to the pair $\mathcal{B}_{a} \subset \mathcal{B}_{\pi}$, and undersampling relative to the pair $\mathcal{B}_{\pi} \subset \mathcal{B}_{b}$ (for dB spaces defined according to (1.6)). These particular choices are related to a convenient simplification in the proofs, but our results can be extended to an arbitrary pair $\mathcal{B}_{a} \subset \mathcal{B}_{b}$ by a scaling argument. Second, the sampling formulae use the spectra of selfadjoint operators with Neumann boundary condition at the left endpoint. This choice simplifies the asymptotic formulae for eigenvalues of the associated Schrödinger operator; it can also be removed but at the expense of a somewhat clumsier analysis. In our opinion this extra workload would not add anything substantial to the results. Finally, and more importantly from our point of view, our assumption on the potential functions is a bit too restrictive. In view of [17], we believe that our results should be valid just requiring $V \in L_{1}(0, s)$, but relaxing our present assumption on $V$ would require some major changes in the details of our proofs. Further generalizations of the results presented here (in particular, involving a wider class of $\mathrm{dB}$ spaces) are the subject of a future work.

About the organization of this work: Sect. 2 recalls the necessary elements on de Branges spaces and regular Schrödinger operators. Section 3 deals with oversampling. Undersampling is treated in Sect. 4. The "Appendix" contains some technical results. 


\section{2 dB Spaces and Schrödinger Operators}

There are various ways of defining a de Branges space (see [4, Sec. 19], [17, Sec. 2], [21]). We recall the following definition: a Hilbert space of entire functions $\mathcal{B}$ is a de Branges ( $\mathrm{dB}$ space) when it has a reproducing kernel $k(z, w)$ and is isometrically invariant under the mappings $f(z) \mapsto f^{\#}(z):=\overline{f(\bar{z})}$ and

$$
f(z) \mapsto\left(\frac{z-\bar{w}}{z-w}\right)^{\operatorname{Ord}_{w}(f)} f(z), \quad w \in \mathbb{C},
$$

where $\operatorname{Ord}_{w}(f)$ is the order of $w$ as a zero of $f$. The class of $\mathrm{dB}$ spaces appearing in this work has the following additional properties:

(a1) Given any real point $x$, there is a function $f \in \mathcal{B}$ such that $f(x) \neq 0$.

(a2) $\mathcal{B}$ is regular, i.e., for any $w \in \mathbb{C}$ and $f \in \mathcal{B},(z-w)^{-1}(f(z)-f(w)) \in \mathcal{B}$.

A distinctive structural property of $\mathrm{dB}$ spaces is that the set of $\mathrm{dB}$ subspaces of a given $\mathrm{dB}$ space is totally ordered by inclusion [4, Thm. 35]. For regular $\mathrm{dB}$ spaces (in the sense of (a2)) this means that, if $\mathcal{B}_{1}$ and $\mathcal{B}_{2}$ are subspaces of a $\mathrm{dB}$ space that are themselves $\mathrm{dB}$ spaces, then either $\mathcal{B}_{1} \subset \mathcal{B}_{2}$ or $\mathcal{B}_{1} \supset \mathcal{B}_{2}$ [6, Sec. 6.5].

The operator $S$ of multiplication by the independent variable in a $\mathrm{dB}$ space $\mathcal{B}$ is defined by

$$
(S f)(z)=z f(z), \quad \operatorname{dom}(S):=\{f \in \mathcal{B}: S f \in \mathcal{B}\} .
$$

This operator is closed, symmetric and has deficiency indices $(1,1)$.

In view of (a1), the spectral core of $S$ is empty (cf. [10, Sec. 4]), i.e., for any $z \in \mathbb{C}$, the operator $(S-z I)^{-1}$ is bounded although, as a consequence of the indices being $(1,1)$, its domain has codimension one. We consider $\mathrm{dB}$ spaces such that $S$ is densely defined and denote by $S(\gamma), \gamma \in[0, \pi)$, the selfadjoint restrictions of $S^{*}$.

Since $\left\langle\left(S^{*}-w\right) k(\cdot, \bar{w}), f(\cdot)\right\rangle=\langle k(\cdot, \bar{w}),(S-\bar{w}) f(\cdot)\rangle=0$ for all $f(z) \in$ $\operatorname{dom}(S)$, we have $k(z, \bar{w}) \in \operatorname{ker}\left(S^{*}-w I\right)$ for any $w \in \mathbb{C}$. Thus

$$
\{k(z, t): t \in \operatorname{spec}(S(\gamma))\} \text { is an orthogonal basis, }
$$

where $\operatorname{spec}(S(\gamma))$ denotes the spectrum of $S(\gamma)$. Hence, the sampling formula

$$
f(z)=\sum_{t \in \operatorname{spec}(S(\gamma))} f(t) \frac{k(z, t)}{k(t, t)}, \quad f \in \mathcal{B},
$$

holds true. The convergence of this series is in the $\mathrm{dB}$ space, which in turn implies uniform convergence in compact subsets of $\mathbb{C}$.

The $\mathrm{dB}$ spaces under consideration in this work are related to symmetric operators arising from regular Schrödinger differential expressions. The construction is similar to the one developed in [17], although there are other ways of generating dB spaces from differential equations of the Sturm-Liouville type [5]. 
Consider a differential expression of the form

$$
\tau:=-\frac{d^{2}}{d x^{2}}+V(x)
$$

where we assume

(v1) $V$ is real-valued and belongs to $L_{1}(0, s)$ for arbitrary $s>0$.

For each $s>0, \tau$ determines a closed symmetric operator $H_{s}$ in $L_{2}(0, s)$,

$$
\begin{aligned}
\operatorname{dom}\left(H_{s}\right) & :=\left\{\varphi \in L_{2}(0, s): \tau \varphi \in L_{2}(0, s), \varphi^{\prime}(0)=\varphi(s)=\varphi^{\prime}(s)=0\right\} \\
H_{s} \varphi & :=\tau \varphi .
\end{aligned}
$$

This operator is known to have deficiency indices $(1,1)$ and empty spectral core, that is,

$$
\left\{z \in \mathbb{C}: \text { there is } C_{z}>0 \text { such that }\left\|\left(H_{S}-z I\right) \varphi\right\| \geq C_{z}\|\varphi\|\right\}=\mathbb{C} .
$$

The selfadjoint extensions of $H_{S}$ are given by

$$
\operatorname{dom}\left(H_{s}(\gamma)\right):=\left\{\begin{array}{c}
\varphi \in L_{2}(0, s): \tau \varphi \in L_{2}(0, s), \\
\varphi^{\prime}(0)=0, \varphi(s) \cos \gamma+\varphi^{\prime}(s) \sin \gamma=0 \\
H_{s}(\gamma) \varphi:=\tau \varphi,
\end{array}\right.
$$

with $\gamma \in[0, \pi)$. Finally, the adjoint operator of $H_{S}$ is

$$
\operatorname{dom}\left(H_{s}^{*}\right):=\left\{\varphi \in L_{2}(0, s): \tau \varphi \in L_{2}(0, s), \varphi^{\prime}(0)=0\right\}, \quad H_{s}^{*} \varphi:=\tau \varphi .
$$

Let $\xi: \mathbb{R}_{+} \times \mathbb{C} \rightarrow \mathbb{C}$ be the solution of the eigenvalue problem

$$
\tau \xi(x, z)=z \xi(x, z), \quad \xi(0, z)=1, \quad \xi^{\prime}(0, z)=0 .
$$

(The derivative is taken with respect to the first argument.) The function $\xi(x, z)$ is real entire for any fixed $x \in \mathbb{R}_{+}\left[13\right.$, Thm. 1.1.1], [22, Thm.9.1]. Also, $\xi(\cdot, z) \in L_{2}(0, s)$ for any $z \in \mathbb{C}$. Using [21, Sec.4] one then establishes that $\xi(\cdot, z)$ is entire as an $L_{2}(0, s)$-valued map. Note that $\xi(\cdot, z)$ depends on the potential $V$ but does not depend on the right endpoint $s$.

According to [19, Props. 2.12 and 2.14] [21, Thm. 16], the functions

$$
f(z)=\langle\xi(\cdot, \bar{z}), \varphi(\cdot)\rangle_{L_{2}(0, s)},
$$

with $\varphi \in L_{2}(0, s)$, form a $\mathrm{dB}$ space $\mathcal{B}_{s}$ with the norm given by

$$
\|f\|_{\mathcal{B}_{s}}=\|\varphi\|_{L_{2}(0, s)} .
$$

A straightforward computation shows that the reproducing kernel of $\mathcal{B}_{s}$ is

$$
k_{s}(z, w)=\langle\xi(\cdot, \bar{z}), \xi(\cdot, \bar{w})\rangle_{L_{2}(0, s)} .
$$


Remark 1 In view of (2.7), $k_{s}(z, w)$ and $\xi(\cdot, \bar{w})$ are related by the isometry (2.5). Hence, using (2.2) and expression (2.6) for the norm in $\mathcal{B}_{s}$, one obtains

$$
\varphi(x)=\sum_{t \in \operatorname{spec}\left(H_{s}(\gamma)\right)} \frac{1}{k_{s}(t, t)}\langle\xi(\cdot, t), \varphi(\cdot)\rangle_{L_{2}(0, s)} \xi(x, t), \quad \varphi \in L_{2}(0, s),
$$

where the series converges in the $L_{2}$-norm.

If $r<s$, then $\mathcal{B}_{r}$ is a proper $\mathrm{dB}$ subspace of $\mathcal{B}_{s}$. Indeed, $\left\{\mathcal{B}_{r}: r \in(0, s)\right\}$ is a chain of $\mathrm{dB}$ subspaces of $\mathcal{B}_{s}$ in accordance with [4, Thm. 35]. The isometry from $L_{2}(0, s)$ onto $\mathcal{B}_{s}$ induced by (2.5) transforms $H_{s}$ into the operator of multiplication by the independent variable in $\mathcal{B}_{s}$ (see (2.1)), the latter will subsequently be denoted by $S_{s}$. Also, the selfadjoint extensions $H_{S}(\gamma)$ are transformed into the selfadjoint extensions $S_{S}(\gamma)$ of $S_{S}$. When referring to unitary invariants (such as the spectrum), we use interchangeably either $H_{S}(\gamma)$ or $S_{S}(\gamma)$ throughout this text.

Remark 2 The space $\mathcal{B}_{s}$ constructed from $L_{2}(0, s)$ via $(2.5)$ depends on the potential $V$, which is assumed to satisfy (v1). However, as shown in [17, Thm. 4.1], the set of entire functions in $\mathcal{B}_{s}$ is the same for all $V \in L_{1}(0, s)$; what changes with $V$ is the inner product in $\mathcal{B}_{S}$. Noteworthily, since the operator $S_{S}$ of multiplication by the independent variable is defined in its maximal domain (see (2.1)), it has always the same domain and range and acts in the same way; yet, by modifying the metric of the space, each $V \in L_{1}(0, s)$ gives rise to a different family of selfadjoint extensions of $S_{S}$. As a consequence, every function in $\mathcal{B}_{S}$ can be sampled by (2.3) using any sequence $\left\{\lambda_{n}\right\}$ as sampling points, as long as there exists $V \in L_{1}(0, s)$ such that $\left\{\lambda_{n}\right\}$ is the spectrum of some selfadjoint extension of the corresponding operator $H_{s}$. This fact can be considered as a generalization of the notion of irregular sampling, quite well studied in Paley-Wiener spaces by means of classical analysis; the Kadec's 1/4 Theorem is a chief example of this kind of results [9].

\section{Oversampling}

The oversampling of a function in $\mathcal{B}_{a}$ is related to the fact that it can be sampled as a function in $\mathcal{B}_{b}$ and the sampling kernel can be modified in such a way that the sampling series is convergent under $l_{\infty}$ perturbations of the samples (see the Sect. 1).

Let $0<a<b<\infty$ and $V$ be as in (v1). Any $\varphi \in L_{2}(0, a)$ can be identified with an element in $L_{2}(0, b)$ since

$$
\varphi=\varphi \chi_{[0, a]}+0 \chi_{(a, b]},
$$

where $\chi_{E}$ denotes the characteristic function of a set $E$. Define

$$
\mathcal{R}(x)=\mathcal{R}_{a b}(x):=\chi_{[0, a]}(x)+\frac{b-x}{b-a} \chi_{(a, b]}(x), \quad x \in[0, b] .
$$


Taking into account (2.8) with $s=b,(3.1)$ and (3.2) imply

$$
\varphi(x)=\sum_{t \in \operatorname{spec}\left(H_{b}(\gamma)\right)} \frac{1}{k_{b}(t, t)}\langle\xi(\cdot, t), \varphi(\cdot)\rangle_{L_{2}(0, b)} \mathcal{R}(x) \xi(x, t),
$$

where the convergence is in $L_{2}(0, b)$. Plugging (3.3) into (2.5) with $s=b$, we obtain

$$
f(z)=\sum_{t \in \operatorname{spec}\left(H_{b}(\gamma)\right)} \frac{1}{k_{b}(t, t)}\langle\xi(\cdot, \bar{z}), \mathcal{R}(\cdot) \xi(\cdot, t)\rangle_{L_{2}(0, b)} f(t), \quad z \in \mathbb{C},
$$

which converges uniformly in compact subsets of $\mathbb{C}$.

Hypothesis 3.1 Given $0<a<b$, the series

$$
\sum_{t \in \operatorname{spec}\left(H_{b}(\gamma)\right)} \frac{1}{k_{b}(t, t)}\left|\left\langle\xi(\cdot, \bar{z}), \mathcal{R}_{a b}(\cdot) \xi(\cdot, t)\right\rangle_{L_{2}(0, b)}\right|
$$

converges uniformly in compact subsets of $\mathbb{C}$.

Assume that Hypothesis 3.1 is met. Enumerate any given sequence $\epsilon \in l_{\infty}$ such

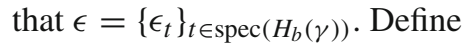

$$
\tilde{\mathcal{K}}_{a b}(z, t):=\frac{1}{k_{b}(t, t)}\left\langle\xi(\cdot, \bar{z}), \mathcal{R}_{a b}(\cdot) \xi(\cdot, t)\right\rangle_{L_{2}(0, b)} .
$$

In view of (3.4), the function

$$
\tilde{f}(z):=\sum_{t \in \operatorname{spec}\left(H_{b}(\gamma)\right)} \tilde{\mathcal{K}}_{a b}(z, t)\left(f(t)+\epsilon_{t}\right), \quad z \in \mathbb{C},
$$

is well defined and the defining series converges uniformly in compact subsets of $\mathbb{C}$. Moreover,

$$
|\tilde{f}(z)-f(z)| \leq\|\epsilon\|_{l_{\infty}} \sum_{t \in \operatorname{spec}\left(H_{b}(\gamma)\right)} \frac{1}{k_{b}(t, t)}\left|\langle\xi(\cdot, \bar{z}), \mathcal{R}(\cdot) \xi(\cdot, t)\rangle_{L_{2}(0, b)}\right|
$$

for all $z \in \mathbb{C}$. Thus, the difference $|\tilde{f}(z)-f(z)|$ is uniformly bounded in compact subsets of $\mathbb{C}$. Below we prove that Hypothesis 3.1 holds true when

(v2) $V$ is real-valued and in $A C[0, b]$ (hence it satisfies (v1) for $s \leq b$ ).

This is performed in two stages, the first one deals with the case $V \equiv 0$, the second one employs perturbative methods to consider the general case.

If $V \equiv 0$, the function $\xi$ given in Sect. 2 is

$$
\xi(x, z)=\cos (\sqrt{z} x), \quad x \in \mathbb{R}_{+} .
$$


Whenever we refer to the function $\xi$ corresponding to $V \equiv 0$, we write the righthand-side of (3.8). We reserve the use of the symbol $\xi$ only for the case $V \not \equiv 0$. Also, throughout this paper we use the main branch of the square root function.

As mentioned in the Sect. 1, for the sake of simplicity we assume $b=\pi$ and fix $\gamma=\pi / 2$. A straightforward calculation yields

$$
\operatorname{spec}\left(H_{\pi}(\pi / 2)\right)=\left\{n^{2}: n \in \mathbb{N} \cup\{0\}\right\}
$$

Moreover, by substituting (3.8) into (2.7), we verify that the reproducing kernel $\stackrel{\circ}{k}_{\pi}(z, w)$ corresponding to the case $V \equiv 0$ satisfies

$$
\stackrel{\circ}{k}_{\pi}\left(n^{2}, n^{2}\right)= \begin{cases}\pi & \text { if } n=0, \\ \frac{\pi}{2} & \text { if } n \in \mathbb{N} .\end{cases}
$$

In the remainder of this section, we denote $\langle\cdot, \cdot\rangle_{L_{2}(0, \pi)}$ simply as $\langle\cdot, \cdot\rangle$.

Proposition 3.2 Hypothesis 3.1 holds true under the assumption $V \equiv 0, b=\pi$, and $\gamma=\pi / 2$.

Proof Consider a compact set $K$ in $\mathbb{C}$ such that $\operatorname{spec}\left(H_{\pi}(\pi / 2)\right)$ intersects $K$ only at $n_{0}^{2}$ with $n_{0} \in \mathbb{N}$. It will be clear at the end of the proof that there is no loss of generality in this assumption. First note that $\left|\left\langle\cos (\sqrt{\bar{z}} \cdot), \mathcal{R}(\cdot) \cos \left(n_{0} \cdot\right)\right\rangle\right|$ is uniformly bounded in $K$ (one can use the Cauchy-Schwarz inequality and note that the factor depending on $z$ is continuous in $K$ ). On the other hand, by Lemma A.5,

$$
\begin{aligned}
& \sum_{n \neq n_{0}}|\langle\cos (\sqrt{z} \cdot), \mathcal{R}(\cdot) \cos (n \cdot)\rangle| \\
& =\frac{1}{2} \sum_{n \neq n_{0}}\left|\frac{\cos ((\sqrt{z}+n) a)-(-1)^{n} \cos (\sqrt{z} \pi)}{(\pi-a)(\sqrt{z}+n)^{2}}+\frac{\cos ((\sqrt{z}-n) a)-(-1)^{n} \cos (\sqrt{z} \pi)}{(\pi-a)(\sqrt{z}-n)^{2}}\right| \\
& \leq \frac{e^{\pi|\operatorname{Im} \sqrt{z}|}}{(\pi-a)} \sum_{n \neq n_{0}}\left(\frac{1}{|\sqrt{z}+n|^{2}}+\frac{1}{|\sqrt{z}-n|^{2}}\right) .
\end{aligned}
$$

Thus, taking into account (3.10), the series (3.5) converges uniformly in $K$.

Now, let us address the case of non-zero $V$ satisfying (v2). As before we set $b=\pi$ and $\gamma=\pi / 2$. Also, we assume $\operatorname{spec}\left(H_{\pi}(\pi / 2)\right)=\left\{\lambda_{n}\right\}_{n=0}^{\infty}$ ordered such that $\lambda_{n-1}<\lambda_{n}$ for all $n \in \mathbb{N}$. The subsequent analysis make use of the following auxiliary functions.

Definition 3.3 For each $x \in[0, \pi], n \in \mathbb{N}$ and $z \in \mathbb{C}$, consider

$$
\begin{aligned}
\rho(x) & :=\frac{1}{2} \int_{0}^{x} V(y) d y-\frac{x}{2 \pi} \int_{0}^{\pi} V(y) d y, \\
T(x, n) & :=\xi\left(x, \lambda_{n}\right)-\cos (n x)-\frac{\rho(x)}{n} \sin (n x), \\
F(x, z) & :=\xi(x, z)-\cos (\sqrt{z} x) .
\end{aligned}
$$


Lemma 3.4 Let $V$ be as in (v2) with $b=\pi$. There exists $N \in \mathbb{N}$ such that, if $n \geq N$, then

$$
\left|\left\langle\xi(\cdot, \bar{z}), \mathcal{R}(\cdot) \xi\left(\cdot, \lambda_{n}\right)\right\rangle-\langle\cos (\sqrt{\bar{z}} \cdot), \mathcal{R}(\cdot) \cos (n \cdot)\rangle\right| \leq C_{\pi} \frac{e^{|\operatorname{Im} \sqrt{z}| \pi}}{n^{2}}\left(1+\frac{1+|z|}{1+\pi|z|^{1 / 2}}\right),
$$

for every $z \in \mathbb{C}$. Here $C_{\pi}$ is a positive number depending on $V$.

Proof In terms of the functions introduced in Definition 3.3, one writes

$$
\begin{aligned}
& \left\langle\xi(\cdot, \bar{z}), \mathcal{R}(\cdot) \xi\left(\cdot, \lambda_{n}\right)\right\rangle-\langle\cos (\sqrt{\bar{z}} \cdot), \mathcal{R}(\cdot) \cos (n \cdot)\rangle \\
& =\int_{0}^{\pi}\left[\cos (\sqrt{z} x) \mathcal{R}(x) \frac{\rho(x)}{n} \sin (n x)+F(x, z) \mathcal{R}(x) \frac{\rho(x)}{n} \sin (n x)\right. \\
& \quad+F(x, z) \mathcal{R}(x) \cos (n x)+\cos (\sqrt{z} x) \mathcal{R}(x) T(x, n) \\
& \quad+F(x, z) \mathcal{R}(x) T(x, n)] d x .
\end{aligned}
$$

It will be shown that each of the five terms on the right-hand side of (3.12) is appropriately bounded. For the first term, one uses the inequality (A.11) of Lemma A.4 and the first inequality of Lemma A.7. The estimate of the second term is obtain by combining (A.12) of Lemma A.4 and the second inequality of Lemma A.7. The third term on the right-hand side of (3.12) is estimated in Lemma A.6.

As regards the fourth and fifth terms in (3.12), one proceeds as follows. From Lemma A.3(ii), it follows that

$$
|T(x, n)| \leq \frac{D}{n^{2}}, \quad D>0,
$$

uniformly with respect to $x \in[0, \pi]$ for $n$ sufficiently large. Also, $|\mathcal{R}(x)| \leq 1$ according to (3.2). Therefore, one has

$$
\left|\int_{0}^{\pi} T(x, n) \mathcal{R}(x) \cos (\sqrt{z} x) d x\right| \leq \frac{C_{1}}{n^{2}} e^{|\operatorname{Im} \sqrt{z}| \pi}
$$

since

$$
|\cos (\sqrt{z} x)| \leq \exp (|\operatorname{Im} \sqrt{z}| \pi), \quad x \in[0, \pi] .
$$

The bound for the remaining term follows by a similar reasoning taking into account (A.4). Thus,

$$
\left|\int_{0}^{\pi} T(x, n) \mathcal{R}(x) F(x, z) d x\right| \leq \frac{C_{2}}{n^{2}} \frac{\pi^{2}}{1+\pi|z|^{1 / 2}} e^{|\operatorname{Im} \sqrt{z}| \pi} .
$$

By combining the estimates of the first three terms, together with (3.13) and (3.14), the bound of the statement is established.

Proposition 3.5 Let $V$ be as in (v2). If $b=\pi$ and $\gamma=\pi / 2$, then Hypothesis 3.1 holds true. 
Proof From Lemma A.3(iii) we know that $k_{\pi}\left(\lambda_{n}, \lambda_{n}\right)-\stackrel{\circ}{k}_{\pi}\left(n^{2}, n^{2}\right)=\mathcal{O}\left(n^{-2}\right)$ as $n \rightarrow \infty$. This implies that

$$
k_{\pi}\left(\lambda_{n}, \lambda_{n}\right) \geq \frac{\stackrel{\circ}{k_{\pi}}\left(n^{2}, n^{2}\right)}{2}=\frac{\pi}{4}
$$

for $n$ sufficiently large, where we have used (3.10). Hence,

$$
\left|\frac{1}{k_{\pi}\left(\lambda_{n}, \lambda_{n}\right)}-\frac{1}{\dot{o}_{\pi}\left(n^{2}, n^{2}\right)}\right|=\frac{\left|k_{\pi}\left(\lambda_{n}, \lambda_{n}\right)-\frac{\pi}{2}\right|}{\frac{\pi}{2} k_{\pi}\left(\lambda_{n}, \lambda_{n}\right)} \leq \frac{8}{\pi^{2}}\left|k_{\pi}\left(\lambda_{n}, \lambda_{n}\right)-\frac{\pi}{2}\right|
$$

for $n$ suficiently large. Again resorting to Lemma A.3(iii), one obtains

$$
\frac{1}{k_{\pi}\left(\lambda_{n}, \lambda_{n}\right)}-\frac{1}{k_{\pi}\left(n^{2}, n^{2}\right)}=\mathcal{O}\left(n^{-2}\right), \quad n \rightarrow \infty .
$$

Due to Lemma 3.4 and (3.15) there exists $N \in \mathbb{N}$ such that, if $n \geq N$, then

$$
\left|\frac{\left\langle\xi(\cdot, \bar{z}), \mathcal{R}(\cdot) \xi\left(\cdot, \lambda_{n}\right)\right\rangle}{k_{\pi}\left(\lambda_{n}, \lambda_{n}\right)}-\frac{\langle\cos (\sqrt{\bar{z}} \cdot), \mathcal{R}(\cdot) \cos (n \cdot)\rangle}{\grave{k}_{\pi}\left(n^{2}, n^{2}\right)}\right| \leq \frac{c_{1}(z)}{n^{2}},
$$

for all $z \in \mathbb{C}$, and where $c_{1}: \mathbb{C} \rightarrow \mathbb{R}$ is a positive continuous function. As a consequence of the previous inequality, there exists another positive continuous function $c_{2}: \mathbb{C} \rightarrow \mathbb{R}$ such that

$$
\sum_{n=0}^{\infty}\left|\frac{\left\langle\xi(\cdot, \bar{z}), \mathcal{R}(\cdot) \xi\left(\cdot, \lambda_{n}\right)\right\rangle}{k_{\pi}\left(\lambda_{n}, \lambda_{n}\right)}-\frac{\langle\cos (\sqrt{\bar{z}} \cdot), \mathcal{R}(\cdot) \cos (n \cdot)\rangle}{\dot{\circ}_{\pi}\left(n^{2}, n^{2}\right)}\right| \leq c_{2}(z) .
$$

Hence, by Proposition 3.2, the series (3.5) converges uniformly in compact subsets of $\mathbb{C}$.

Arguing as in the paragraph below Hypothesis 3.1, one arrives at the following assertion in which the oversampling procedure is established (see the Sect. 1).

Theorem 3.6 Suppose $V$ obeys (v2) with $b=\pi$. Consider $\mathcal{B}_{a}$ with $a \in(0, \pi)$. Then, for every compact set $K \subset \mathbb{C}$, there exist a constant $C(a, K, V)>0$ such that

$$
|f(z)-\tilde{f}(z)| \leq C(a, K, V)\|\epsilon\|_{\infty}, \quad z \in K,
$$

for all $f(z) \in \mathcal{B}_{a}$, where $\epsilon=\left\{\epsilon_{t}\right\}$ is any bounded real sequence and $\tilde{f}(z)$ is given by (3.7) with $b=\pi$ and $\gamma=\pi / 2$. 


\section{Undersampling}

In this section, we treat undersampling of functions in $\mathcal{B}_{b} \backslash \mathcal{B}_{a}(a<b)$ with the sampling points given by the spectrum of $S_{a}(\gamma)$ as explained in the Sect. 1 .

Hypothesis 4.1 For $a<b$ and each $z \in \mathbb{C}$, the series

$$
\sum_{t \in \operatorname{spec}\left(H_{a}(\gamma)\right)} \frac{k_{a}(t, \bar{z})}{k_{a}(t, t)} \xi(x, t)
$$

converges absolutely and uniformly with respect to $x \in[0, b]$.

Remark 3 Note that (2.7) and (2.8) imply that the series

$$
\sum_{t \in \operatorname{spec}\left(H_{a}(\gamma)\right)} \frac{k_{a}(t, \bar{z})}{k_{a}(t, t)} \xi(\cdot, t)
$$

converges to $\xi(\cdot, z)$ in $L_{2}(0, a)$ for each $z \in \mathbb{C}$. Due to (2.2), if $z=\lambda \in \operatorname{spec}\left(H_{a}(\gamma)\right)$, then $k_{a}(t, \lambda)=0$ for $t \in \operatorname{spec}\left(H_{a}(\gamma)\right) \backslash\{\lambda\}$. in which case the series (4.2) and (4.1) have only one term.

Lemma 4.2 Assume that Hypothesis 4.1 is met. Define

$$
\xi_{a}^{e x t}(x, z):=\sum_{t \in \operatorname{spec}\left(H_{a}(\gamma)\right)} \frac{k_{a}(t, \bar{z})}{k_{a}(t, t)} \xi(x, t), \quad x \in[0, b], \quad z \in \mathbb{C} .
$$

Then, for each $z \in \mathbb{C}$,

(i) $\xi_{a}^{\text {ext }}(\cdot, z)$ is continuous in $[0, b]$,

(ii) $\xi_{a}^{\text {ext }}(x, z)=\xi(x, z)$ for a. e. $x \in[0, a]$, and

(iii) the function $h_{a}(z):=\sup _{x \in[a, b]}\left|\xi_{a}^{e x t}(x, z)-\xi(x, z)\right|$ is continuous in $\mathbb{C}$.

Moreover,

(iv) if $\psi \in L_{2}(0, b)$ and $g(z) \in \mathcal{B}_{b}$ are related by the isometry (2.5), then

$$
\left\langle\xi_{a}^{\text {ext }}(\cdot, \bar{z}), \psi(\cdot)\right\rangle_{L_{2}(0, b)}=\sum_{t \in \operatorname{spec}\left(H_{a}(\gamma)\right)} \frac{k_{a}(t, \bar{z})}{k_{a}(t, t)} g(t), \quad z \in \mathbb{C} .
$$

Proof Enumerate spec $\left(H_{a}(\gamma)\right)=\left\{\lambda_{n}\right\}_{n=0}^{\infty}$ such that $\lambda_{n-1}<\lambda_{n}$ for all $n \in \mathbb{N}$. Then (i) is a straightforward consequence of Hypothesis 4.1. Due to (i), $\xi_{a}^{\text {ext }}(\cdot, z)$ is an element of $L_{2}(0, a)$ for each $z \in \mathbb{C}$. Thus, Hypothesis 4.1 implies

$$
\lim _{m \rightarrow \infty}\left\|\xi_{a}^{e x t}(\cdot, z)-\sum_{n=0}^{m} \frac{k_{a}\left(\lambda_{n}, \bar{z}\right)}{k_{a}\left(\lambda_{n}, \lambda_{n}\right)} \xi\left(\cdot, \lambda_{n}\right)\right\|_{L_{2}(0, a)}=0 .
$$


This, along with Remark 3, yields (ii). Item (iii) follows from Lemma A.1. To prove (iv), apply the dominated convergence theorem, which holds because of Hypothesis 4.1,

$$
\left\langle\xi_{a}^{e x t}(\cdot, \bar{z}), \psi(\cdot)\right\rangle_{L_{2}(0, b)}=\lim _{m \rightarrow \infty} \sum_{n=0}^{m} \frac{k_{a}\left(\lambda_{n}, \bar{z}\right)}{k_{a}\left(\lambda_{n}, \lambda_{n}\right)} \int_{0}^{b} \xi\left(x, \lambda_{n}\right) \psi(x) d x .
$$

Assume that Hypothesis 4.1 holds true. Suppose that $\psi \in L_{2}(0, b)$ and $g(z) \in \mathcal{B}_{b}$ are related by the isometry $(2.5)$, that is,

$$
g(z)=\langle\xi(\cdot, \bar{z}), \psi(\cdot)\rangle_{L_{2}(0, b)}, \quad z \in \mathbb{C}
$$

Define

$$
\widehat{g}(z):=\left\langle\xi_{a}^{e x t}(\cdot, \bar{z}), \psi(\cdot)\right\rangle_{L_{2}(0, b)}, \quad z \in \mathbb{C} .
$$

Then, due to Lemma 4.2(ii),

$$
|g(z)-\widehat{g}(z)|=\left|\int_{a}^{b}\left(\xi(x, z)-\xi_{a}^{e x t}(x, z)\right) \psi(x) d x\right| \leq h_{a}(z) \int_{a}^{b}|\psi(x)| d x,
$$

where the function $h_{a}$ has been defined in Lemma 4.2(iii). Therefore, for each $\psi \in$ $L_{2}(0, b)$, the difference $|g(z)-\widehat{g}(z)|$ is uniformly bounded in compact subsets of $\mathbb{C}$. Below we prove that Hypothesis 4.1 holds true when $V$ satisfies (v2) with $b>\pi$. As in the previous section, this is performed in two stages, the first one deals with the particular case $V \equiv 0$ and the second one treats the general case.

In keeping with the simplification made in the previous section, we consider only the case $a=\pi$ and $\gamma=\pi / 2$.

Using trigonometric identities and Eqs. (2.7) and (3.8) one verifies that

$$
\stackrel{\circ}{k}_{\pi}\left(n^{2}, \bar{z}\right)=\int_{0}^{\pi} \cos (n x) \cos (\sqrt{z} x) d x=\frac{(-1)^{n+1}}{n^{2}-z} \sqrt{z} \sin (\sqrt{z} \pi) .
$$

whenever $n \in \mathbb{N} \cup\{0\}$ and $z \in \mathbb{C} \backslash\left\{n^{2}\right\}$. Recall that $\stackrel{\circ}{\pi}_{\pi}$ denotes the reproducing kernel within $\mathcal{B}_{\pi}$ associated with $V \equiv 0$.

Proposition 4.3 Hypothesis 4.1 holds true under the assumption $V \equiv 0, a=\pi$, and $\gamma=\pi / 2$.

Proof Let $K$ be a compact subset of $\mathbb{C}$. As in the proof of Proposition 3.2, assume without loss of generality that $n_{0}^{2}$ is the only point of $\operatorname{spec}\left(H_{\pi}(\pi / 2)\right)$ in $K\left(n_{0} \in\right.$ $\mathbb{N})$. Due to (3.8)-(3.10), it suffices to show the uniform convergence of the series $\sum_{n \neq n_{0}}\left|\stackrel{\circ}{\pi}_{\pi}\left(n^{2}, \bar{z}\right)\right|$ in $K$. By (4.6), one obtains

$$
\sum_{n \neq n_{0}}\left|\grave{k}_{\pi}\left(n^{2}, \bar{z}\right)\right| \leq|\sqrt{z} \sin (\sqrt{z} \pi)| \sum_{n \neq n_{0}} \frac{1}{\left|n^{2}-z\right|}
$$


Now we address the case of nontrivial potential $V$ satisfying (v2) with $b>\pi$. Let $\operatorname{spec}\left(H_{\pi}(\pi / 2)\right)=\left\{\lambda_{n}\right\}_{n=0}^{\infty}$ such that $\lambda_{n-1}<\lambda_{n}$ for all $n \in \mathbb{N}$. We aim to study the difference

$$
\frac{k_{\pi}\left(\lambda_{n}, \bar{z}\right)}{k_{\pi}\left(\lambda_{n}, \lambda_{n}\right)} \xi\left(x, \lambda_{n}\right)-\frac{\stackrel{\circ}{k}_{\pi}\left(n^{2}, \bar{z}\right)}{\dot{\circ}_{\pi}\left(n^{2}, n^{2}\right)} \cos (n x), \quad x \in[0, b], \quad z \in \mathbb{C},
$$

for any given $b>\pi$ and all $n \in \mathbb{N}$ large enough.

Lemma 4.4 For any $V$ satisfying (v2) with $b>\pi$, there exists an $N \in \mathbb{N}$ such that, if $n \geq N$, then

$$
\left|k_{\pi}\left(\lambda_{n}, \bar{z}\right)-\stackrel{\circ}{k}_{\pi}\left(n^{2}, \bar{z}\right)\right| \leq D_{\pi} \frac{e^{|\operatorname{Im} \sqrt{z}| \pi}}{n^{2}}\left(1+\frac{1+|z|}{1+\pi|z|^{1 / 2}}\right),
$$

for every $z \in \mathbb{C}$. Here $D_{\pi}$ is a positive real number depending on $V$.

Proof In view of (2.7) and Definition 3.3,

$$
\begin{aligned}
k_{\pi}\left(\lambda_{n}, \bar{z}\right) & -\stackrel{\circ}{k}_{\pi}\left(n^{2}, \bar{z}\right)=\int_{0}^{\pi}\left[\cos (n x) F(x, z)+\frac{\rho(x)}{n} \sin (n x) \cos (\sqrt{z} x)\right. \\
& \left.+\frac{\rho(x)}{n} \sin (n x) F(x, z)+T(x, n) \cos (\sqrt{z} x)+T(x, n) F(x, z)\right] d x .
\end{aligned}
$$

We proceed as in the proof of Lemma 3.4. The first three terms on the right-hand side of the last equality are estimated by Lemma A.4. The remaining terms have estimates obtained in the same way as the estimates (3.13) and (3.14).

Lemma 4.5 Assume that $V$ satisfies (v2) and $b>\pi$. Then, the asymptotic formula

$$
\xi\left(x, \lambda_{n}\right)-\cos (n x)=\mathcal{O}\left(n^{-1}\right), \quad n \rightarrow \infty,
$$

holds uniformly with respect to $x \in[0, b]$.

Proof Using Lemma A.3(i) and repeating the reasoning leading to (3.15), one arrives at

$$
\lambda_{n}^{-1 / 2}-n^{-1}=\mathcal{O}\left(n^{-1}\right), \quad n \rightarrow \infty .
$$

This asymptotic formula and (A.4) yield

$$
\xi\left(x, \lambda_{n}\right)-\cos \left(\sqrt{\lambda_{n}} x\right)=\mathcal{O}\left(n^{-1}\right), \quad n \rightarrow \infty .
$$

Finally, since

$$
\left|\cos \left(\sqrt{\lambda_{n}} x\right)-\cos (n x)\right|=\left|\sin \left(\alpha_{n} x\right)\right|\left|\sqrt{\lambda_{n}} x-n x\right| \leq\left|\sqrt{\lambda_{n}}-n\right| b
$$

for some $\alpha_{n}$ between $\sqrt{\lambda_{n}}$ and $n$, the statement follows from Lemma A.3(i). 
Proposition 4.6 Let $V$ be as in (v2) with $b>\pi$. Set $a=\pi$ and $\gamma=\pi / 2$. Then, Hypothesis 4.1 holds true.

Proof Due to Lemmas 4.4 and 4.5, along with (3.15), there exists $N \in \mathbb{N}$ and a continuous positive function $c_{3}: \mathbb{C} \rightarrow \mathbb{R}$ such that

$$
\left|\frac{k_{\pi}\left(\lambda_{n}, \bar{z}\right)}{k_{\pi}\left(\lambda_{n}, \lambda_{n}\right)} \xi\left(x, \lambda_{n}\right)-\frac{\stackrel{\circ}{k}_{\pi}\left(n^{2}, \bar{z}\right)}{\dot{\circ}_{\pi}\left(n^{2}, n^{2}\right)} \cos (n x)\right| \leq \frac{c_{3}(z)}{n^{2}}, \quad z \in \mathbb{C}, \quad x \in[0, b] .
$$

for all $n \geq N$; we note that $c_{3}$ may depend on $b$ and $V$. The estimate (4.7) in turn implies

$$
\sum_{n=0}^{\infty}\left|\frac{k_{\pi}\left(\lambda_{n}, \bar{z}\right)}{k_{\pi}\left(\lambda_{n}, \lambda_{n}\right)} \xi\left(x, \lambda_{n}\right)-\frac{\stackrel{\circ}{k}_{\pi}\left(n^{2}, \bar{z}\right)}{\dot{k}_{\pi}\left(n^{2}, n^{2}\right)} \cos (n x)\right| \leq c_{4}(z)
$$

uniformly with respect to $x \in[0, b]$, where $c_{4}: \mathbb{C} \rightarrow \mathbb{R}$ is another continuous positive function that may also depend on $b$ and $V$. The claimed assertion now follows from Proposition 4.3.

Theorem 4.7 Suppose $V$ obeys (v2) for $b>\pi$. Assume that $\psi \in L_{2}(0, b)$ and $g(z) \in$ $\mathcal{B}_{b}$ are related by (4.4). For every compact $K \subset \mathbb{C}$, there exist a constant $D(b, K, V)>$ 0 such that

$$
|g(z)-\widehat{g}(z)| \leq D(b, K, V) \int_{\pi}^{b}|\psi(x)| d x, \quad z \in K,
$$

where $\widehat{g}(z)$ is given by (4.5) with $a=\pi$, i.e., $\widehat{g}(z)$ is given by the series (4.3) with $a=\pi$ and $\gamma=\pi / 2$.

Acknowledgements The authors thank the anonymous referee whose pertinent comments led to an improved presentation of this work.

Open Access This article is distributed under the terms of the Creative Commons Attribution 4.0 International License (http://creativecommons.org/licenses/by/4.0/), which permits unrestricted use, distribution, and reproduction in any medium, provided you give appropriate credit to the original author(s) and the source, provide a link to the Creative Commons license, and indicate if changes were made.

\section{Appendix A: Auxiliary results}

Lemma A.1 Let $Y$ be a compact interval of $\mathbb{R}$. Suppose $\theta: \mathbb{C} \times Y \rightarrow[0, \infty)$ is continuous. Then, $\Theta: \mathbb{C} \rightarrow[0, \infty)$ given by $\Theta(z):=\sup \{\theta(z, y): y \in Y\}$ is continuous.

Proof For each $z \in \mathbb{C}$, fix $\vartheta(z) \in Y$ such that

$$
\theta(z, \vartheta(z))=\sup \{\theta(z, y): y \in Y\}=\Theta(z) .
$$

Take an arbitrary $z_{0} \in \mathbb{C}$. Fix $r_{0}>0$ and let $K:=\left\{w \in \mathbb{C}:\left|z_{0}-w\right| \leq r_{0}\right\}$. Due to the compactness of $K \times Y$, the map $\theta \uparrow_{K \times Y}$ is uniformly continuous. Hence, given $\epsilon>0$ there exists $\delta>0$ such that 


$$
|z-w|<\delta \text { and }|y-v|<\delta \text { imply }|\theta(z, y)-\theta(w, v)|<\frac{\epsilon}{2},
$$

for any $(z, y),(w, v) \in K \times Y$. Take $w \in K$ such that $\left|z_{0}-w\right|<\delta$. If $v \in Y$ satisfies $\left|\vartheta\left(z_{0}\right)-v\right|<\delta$ then, in view of (A.2),

$$
\left|\theta\left(z_{0}, \vartheta\left(z_{0}\right)\right)\right|-|\theta(w, v)| \leq\left|\theta\left(z_{0}, \vartheta\left(z_{0}\right)\right)-\theta(w, v)\right|<\frac{\epsilon}{2} .
$$

Due to (A.1) and the fact that $\theta$ is non negative, $\Theta\left(z_{0}\right)-\Theta(w) \leq \Theta\left(z_{0}\right)-\theta(w, v)<\epsilon$. Now, let $v \in Y$ such that $|\vartheta(w)-v|<\delta$. According to (A.2),

$$
|\theta(w, \vartheta(w))|-\left|\theta\left(z_{0}, v\right)\right| \leq\left|\theta(w, \vartheta(w))-\theta\left(z_{0}, v\right)\right|<\frac{\epsilon}{2} .
$$

Hence, $\Theta(w)-\Theta\left(z_{0}\right) \leq \Theta(w)-\theta\left(z_{0}, v\right)<\epsilon$. Therefore, we have proven that $-\epsilon<\Theta\left(z_{0}\right)-\Theta(w)<\epsilon$ whenever $\left|z_{0}-w\right|<\delta$.

The following Lemma is the analogue of [11, Lemma 2.2] for Neumann-like boundary conditions.

Lemma A.2 Given $a>0$, suppose that $V \in L_{1}(0, a)$. Then, for each $z \in \mathbb{C}$, the unique solution of the initial value problem

$$
\begin{aligned}
& -\xi^{\prime \prime}(x, z)+V(x) \xi(x, z)=z \xi(x, z), \quad 0 \leq x \leq a, \\
& \xi(0, z)=1, \quad \xi^{\prime}(0, z)=0,
\end{aligned}
$$

satisfies the integral equation

$$
\xi(x, z)=\cos (\sqrt{z} x)+\int_{0}^{x} G(z, x, y) V(y) \xi(y, z) d y,
$$

where

$$
G(z, x, y)=\frac{1}{\sqrt{z}} \sin (\sqrt{z}(x-y))
$$

is the corresponding Green's function. This solution satisfies the estimate

$$
|\xi(x, z)-\cos (\sqrt{z} x)| \leq C \frac{x}{1+|z|^{1 / 2} x} e^{|\operatorname{Im} \sqrt{z}| x} \int_{0}^{x} \frac{y|V(y)|}{1+|z|^{1 / 2} y} d y
$$

for some constant $C=C(a, V)>0$. Furthermore, the derivative obeys

$$
\xi^{\prime}(x, z)=-\sqrt{z} \sin (\sqrt{z} x)+\int_{0}^{x} \frac{\partial}{\partial x} G(z, x, y) V(y) \xi(y, z) d y,
$$

and satisfies the estimate

$$
\left|\xi^{\prime}(x, z)+\sqrt{z} \sin (\sqrt{z} x)\right| \leq C e^{|\operatorname{Im} \sqrt{z}| x} \int_{0}^{x}|V(y)| d y .
$$




\section{Proof Define}

$$
\xi_{0}(x, z):=\cos (\sqrt{z} x), \quad \xi_{n+1}(x, z):=\int_{0}^{x} G(z, x, y) V(y) \xi_{n}(y, z) d y, \quad n \in \mathbb{N}
$$

Since $|\cos (\sqrt{z} x)| \leq \exp (|\operatorname{Im} \sqrt{z}| x)$ and

$$
|G(z, x, y)| \leq C_{0} \frac{x}{1+|z|^{1 / 2} x} e^{|\operatorname{Im} \sqrt{z}|(x-y)}, \quad 0 \leq y \leq x,
$$

for some constant $C_{0}>0$ (cf. [11, Lemma A.1]), one has

$$
\left|\xi_{1}(x, z)\right| \leq C_{0}\|V\|_{L_{1}} \frac{x}{1+|z|^{1 / 2} x} e^{|\operatorname{Im} \sqrt{z}| x}
$$

An induction argument then shows

$$
\left|\xi_{n+1}(x, z)\right| \leq \frac{\|V\|_{L_{1}} C_{0}^{n+1}}{(n+1) !} \frac{x}{1+|z|^{1 / 2} x} e^{|\operatorname{Im} \sqrt{z}| x}\left(\int_{0}^{x} \frac{y|V(y)|}{1+|z|^{1 / 2} y} d y\right)^{n}
$$

for all $n \in \mathbb{N}$. It follows that

$$
\xi(x, z):=\sum_{n=0}^{\infty} \xi_{n}(x, z)
$$

converges uniformly with respect to $x \in[0, a]$ for all $z \in \mathbb{C}$ and satisfies (A.3). The estimate (A.4) readily follows from (A.7) after noticing that

$$
\int_{0}^{x} \frac{y|V(y)|}{1+|z|^{1 / 2} y} d y \leq a\|V\|_{L_{1}} .
$$

The assertions (A.5) and (A.6) are proved by similar arguments so we omit the details.

The next results refer to the functions $\rho, T$, and $F$ introduced in Definition 3.3, as well as the reproducing kernel $k_{b}(z, w)$ from $(2.7)$ and the particular case $k_{b}(z, w)$ when $V \equiv 0$.

Lemma A.3 Assume that $V$ satisfies ( 22$)$ with $b=\pi$. Let $H_{\pi}(\pi / 2)$ be the selfadjoint operator defined in accordance with (2.4). Enumerate $\operatorname{spec}\left(H_{\pi}(\pi / 2)\right)$ in increasing order and denote $\operatorname{spec}\left(H_{\pi}(\pi / 2)\right)=\left\{\lambda_{n}\right\}_{n=0}^{\infty}$. Then, the following assertions hold true.

(i) $\sqrt{\lambda_{n}}=n+\mathcal{O}\left(n^{-1}\right)$ as $n \rightarrow \infty$,

(ii) $T(x, n)=\mathcal{O}\left(n^{-2}\right)$ as $n \rightarrow \infty$, uniformly with respect to $x \in[0, \pi]$,

(iii) $k_{\pi}\left(\lambda_{n}, \lambda_{n}\right)=\dot{k}_{\pi}\left(n^{2}, n^{2}\right)+\mathcal{O}\left(n^{-2}\right)$ as $n \rightarrow \infty$. 
Proof Items (i) and (ii) are shown in [13, Sec.1.2.2]. We note that the asymptotic formulae in [13] are obtained assuming that $V^{\prime}$ is bounded in $[0, \pi]$. However, one can see that it suffices to require $V^{\prime} \in L_{1}(0, \pi)$.

We turn to the proof of (iii). Let us recall that

$$
k_{\pi}\left(\lambda_{n}, \lambda_{n}\right)=\left\langle\xi\left(\cdot, \lambda_{n}\right), \xi\left(\cdot, \lambda_{n}\right)\right\rangle_{L_{2}(0, \pi)}=\int_{o}^{\pi}\left|\xi\left(x, \lambda_{n}\right)\right|^{2} d x=\int_{o}^{\pi} \xi^{2}\left(x, \lambda_{n}\right) d x
$$

while

$$
\stackrel{\circ}{k}_{\pi}\left(n^{2}, n^{2}\right)=\int_{0}^{\pi}(\cos n x)^{2} d x .
$$

A straightforward computation shows that

$$
\sup _{0 \leq x \leq \pi}|\rho(x)| \leq\|V\|_{L_{1}(0, \pi)}, \quad \sup _{0 \leq x \leq \pi}\left|\rho^{\prime}(x)\right| \leq\|V\|_{L_{1}(0, \pi)} .
$$

Together with (3.11) and (ii), these inequalities imply

$$
\xi^{2}\left(x, \lambda_{n}\right)=(\cos n x)^{2}+\frac{\rho(x)}{n} \sin (2 n x)+\mathcal{O}\left(n^{-2}\right), \quad n \rightarrow \infty
$$

uniformly with respect to $x \in[0, \pi]$. Using integration by parts along with the fact that $\rho(0)=\rho(\pi)=0$, one obtains

$$
\left|\int_{0}^{\pi} \rho(x) \sin (2 n x) d x\right| \leq \frac{1}{2 n} \int_{0}^{\pi}\left|\rho^{\prime}(x) \cos (2 n x)\right| d x .
$$

Assertion (iii) follows from (A.8) and (A.9).

Lemma A.4 Assume $V$ satisfies the hypothesis of Lemma A.3. Consider an arbitrary $a \in(0, \pi]$. Then, for all $z \in \mathbb{C}$ and $n \in \mathbb{N}$, the following inequalities hold true:

$$
\begin{aligned}
\left|\int_{0}^{\pi} F(x, z) \cos (n x) d x\right| & \leq C_{1} \frac{e^{\pi|\operatorname{Im} \sqrt{z}|}}{n^{2}}\left(1+\frac{1+|z|}{1+\pi|z|^{1 / 2}}\right), \\
\left|\int_{0}^{a} \rho(x) \cos (\sqrt{z} x) \sin (n x) d x\right| & \leq C_{2} \frac{e^{\pi|\operatorname{Im} \sqrt{z}|}}{n}\left(1+\frac{|z|}{1+\pi|z|^{1 / 2}}\right), \\
\left|\int_{0}^{a} \rho(x) F(x, z) \sin (n x) d x\right| & \leq C_{3} \frac{e^{\pi|\operatorname{Im} \sqrt{z}|}}{n}\left(1+\frac{1}{1+|z|^{1 / 2} \pi}\right) .
\end{aligned}
$$

Here, $C_{1}>0$ depends on $V$ while $C_{2}>0$ and $C_{3}>0$ may, in addition, depend on $a$.

Proof Integrating by parts one obtains,

$$
\left|\int_{0}^{\pi} F(x, z) \cos (n x) d x\right| \leq \frac{1}{n^{2}}\left(2 \sup _{x \in[0, \pi]}\left|F^{\prime}(x, z)\right|+\pi \sup _{x \in[0, \pi]}\left|F^{\prime \prime}(x, z)\right|\right) .
$$


On one hand, due to (A.6),

$$
\sup _{x \in[0, \pi]}\left|F^{\prime}(x, z)\right| \leq C_{V} \exp (|\operatorname{Im} \sqrt{z}| \pi)
$$

On the other hand, since $F^{\prime \prime}(x, z)=V(x) \xi(x, z)-z F(x, z)$, it follows from (A.4) that

$$
\left|F^{\prime \prime}(x, z)\right| \leq e^{\pi|\operatorname{Im} \sqrt{z}|}\left(\frac{C_{V} x}{1+|z|^{1 / 2} x}\left(\|V\|_{L_{1}}+|z|\right)+\|V\|_{L_{1}}\right) .
$$

This implies (A.10).

The proof of (A.11) repeats the argumentation above: integrate by parts and observe that

$$
\begin{aligned}
\sup _{x \in[0, a]}|\rho(x) \cos (\sqrt{z} x)| & \leq\|V\|_{L_{1}} e^{\pi|\operatorname{Im} \sqrt{z}|}, \\
\sup _{x \in[0, a]}\left|\frac{d}{d x} \rho(x) \cos (\sqrt{z} x)\right| & \leq\|V\|_{L_{1}} e^{\pi|\operatorname{Im} \sqrt{z}|}\left(\frac{|z| C \pi}{1+|z|^{1 / 2} \pi}+1\right) .
\end{aligned}
$$

The proof of (A.12) follows a similar reasoning.

Lemma A.5 Set $a \in(0, \pi)$ and consider $\mathcal{R}_{a \pi}$ given by (3.2). Then, for any $n \in \mathbb{N} \cup\{0\}$ and $z \in \mathbb{C} \backslash\left\{n^{2}\right\}$,

$$
\begin{aligned}
\left\langle\cos (\sqrt{\bar{z}} \cdot), \mathcal{R}_{a \pi}(\cdot) \cos (n \cdot)\right\rangle= & \frac{1}{2(\pi-a)}\left(\frac{\cos ((\sqrt{z}+n) a)-(-1)^{n} \cos (\sqrt{z} \pi)}{(\sqrt{z}+n)^{2}}\right. \\
& \left.+\frac{\cos ((\sqrt{z}-n) a)-(-1)^{n} \cos (\sqrt{z} \pi)}{(\sqrt{z}-n)^{2}}\right) .
\end{aligned}
$$

Proof On one hand, the identity

$$
\cos (\sqrt{z} x) \cos (n x)=2^{-1}(\cos ((\sqrt{z}+n) x)+\cos ((\sqrt{z}-n) x))
$$

leads to

$$
\int_{0}^{a} \cos (\sqrt{z} x) \cos (n x) d x=\frac{1}{2}\left(\frac{\sin ((\sqrt{z}+n) a)}{\sqrt{z}+n}+\frac{\sin ((\sqrt{z}-n) a)}{\sqrt{z}-n}\right) .
$$

On the other hand,

$$
\begin{aligned}
& \int_{a}^{\pi} \cos (\sqrt{z} x) \cos (n x)\left(\frac{\pi-x}{\pi-a}\right) d x \\
& =-\frac{1}{2(\pi-a)}\left(\int_{a}^{\pi} x \cos ((\sqrt{z}+n) x) d x+\int_{a}^{\pi} x \cos ((\sqrt{z}-n) x) d x\right) \\
& \quad+\left.\frac{\pi}{2(\pi-a)}\left(\frac{\sin ((\sqrt{z}+n) x)}{\sqrt{z}+n}+\frac{\sin ((\sqrt{z}-n) x)}{\sqrt{z}-n}\right)\right|_{x=a} ^{x=\pi} .
\end{aligned}
$$


Another integration by parts yields

$$
\begin{aligned}
& \int_{a}^{\pi} x \cos ((\sqrt{z} \pm n) x) d x \\
& \quad=\frac{(-1)^{n} \cos (\sqrt{z} \pi)-\cos ((\sqrt{z} \pm n) a)}{(\sqrt{z} \pm n)^{2}}+\frac{(-1)^{n} \sin (\sqrt{z} \pi)-a \sin ((\sqrt{z} \pm n) a)}{\sqrt{z} \pm n} .
\end{aligned}
$$

This completes the proof.

Lemma A.6 Set $a \in(0, \pi)$ and consider $\mathcal{R}_{a \pi}$ given by (3.2). Then, for every $z \in \mathbb{C}$ and $n \in \mathbb{N}$,

$$
\left|\int_{0}^{\pi} F(x, z) \mathcal{R}_{a \pi}(x) \cos (n x) d x\right| \leq C \frac{e^{\pi|\operatorname{Im} \sqrt{z}|}}{n^{2}}\left(1+\frac{1+|z|}{1+\pi|z|^{1 / 2}}\right),
$$

where $C>0$ may depend on $V$.

Proof Integration by parts yields

$$
\begin{aligned}
& \int_{0}^{a} F(x, z) \cos (n x) d x=\frac{1}{n}\left(F(a, z) \sin (n a)-\int_{0}^{a} F^{\prime}(x, z) \sin (n x) d x\right), \\
& \int_{a}^{\pi} F(x, z) \cos (n x) d x=-\frac{1}{n}\left(F(a, z) \sin (n a)+\int_{a}^{\pi} F^{\prime}(x, z) \sin (n x) d x\right),
\end{aligned}
$$

and

$$
\begin{aligned}
\int_{a}^{\pi} x F(x, z) \cos (n x) d x=- & \frac{1}{n}\left(a F(a, z) \sin (n a)+\int_{a}^{\pi} x F^{\prime}(x, z) \sin (n x) d x\right. \\
& \left.+\int_{a}^{\pi} F(x, z) \sin (n x) d x\right) .
\end{aligned}
$$

Now, (A.14) and (A.15) imply

$$
\begin{aligned}
\int_{a}^{\pi} & F(x, z)\left(\frac{\pi-x}{\pi-a}\right) \cos (n x) d x \\
= & -\frac{\pi}{(\pi-a) n}\left(F(a, z) \sin (n a)+\int_{a}^{\pi} F^{\prime}(x, z) \sin (n x) d x\right) \\
& +\frac{1}{(\pi-a) n}\left(a F(a, z) \sin (n a)+\int_{a}^{\pi} x F^{\prime}(x, z) \sin (n x) d x\right. \\
& \left.+\int_{a}^{\pi} F(x, z) \sin (n x) d x\right) .
\end{aligned}
$$


Then, (A.13) and (A.16) yield

$$
\begin{aligned}
& \int_{0}^{\pi} F(x, z) \mathcal{R}(x) \cos (n x) d x \\
& =-\frac{1}{n} \int_{0}^{a} F^{\prime}(x, z) \sin (n x) d x-\frac{\pi}{(\pi-a) n} \int_{a}^{\pi} F^{\prime}(x, z) \sin (n x) d x \\
& \quad+\frac{1}{(\pi-a) n}\left(\int_{a}^{\pi} x F^{\prime}(x, z) \sin (n x) d x+\int_{a}^{\pi} F(x, z) \sin (n x) d x\right) .
\end{aligned}
$$

The claimed assertion now follows by an argument similar to the proof of Lemma A.4.

Lemma A.7 Let $V$ be as in (v2) with $b=\pi$. Fix $a \in(0, \pi)$. Then,

$$
\left|\int_{a}^{\pi} \cos (\sqrt{z} x)\left(\frac{\pi-x}{\pi-a}\right) \rho(x) \sin (n x) d x\right| \leq \frac{C}{n} e^{\pi|\operatorname{Im} \sqrt{z}|}\left(1+\frac{|z|}{1+\pi|z|^{1 / 2}}\right),
$$

and

$$
\left|\int_{a}^{\pi} F(x, z)\left(\frac{\pi-x}{\pi-a}\right) \rho(x) \sin (n x) d x\right| \leq \frac{C}{n} e^{\pi|\operatorname{Im} \sqrt{z}|}\left(1+\frac{1}{1+\pi|z|^{1 / 2}}\right),
$$

for arbitrary $z \in \mathbb{C}$ and $n \in \mathbb{N}$.

Proof We prove the first inequality. The second one is proved analogously. Arguing as in the beginning of the proof of Lemma A.4, one obtains

$$
\left|\int_{a}^{\pi} \cos (\sqrt{z} x)\left(\frac{\pi-x}{\pi-a}\right) \rho(x) \sin (n x) d x\right| \leq \frac{1}{n}\left(2 M_{1}(z)+\pi M_{2}(z)\right),
$$

where

$$
M_{1}(z):=\sup \left\{\left|\cos (\sqrt{z} x) \frac{\pi-x}{\pi-a} \rho(x)\right|: x \in[a, \pi]\right\}
$$

and

$$
M_{2}(z):=\sup \left\{\left|\frac{d}{d x}\left(\cos (\sqrt{z} x) \frac{\pi-x}{\pi-a} \rho(x)\right)\right|: x \in[a, \pi]\right\} .
$$

\section{References}

1. Bailey, B.A., Madych, W.R.: Cardinal sine series, oversampling, and periodic distributions. Proc. Am. Math. Soc. 143(10), 4373-4382 (2015)

2. Bailey, B.A., Madych, W.R.: Classical sampling series of band limited functions: oversampling and non-existence. Sampl. Theory Signal Image Process. 15, 131-138 (2016)

3. Bodmann, B.G., Liner, C.: Spikes, roots, and aliasing: recovering bandlimited signals from roots of the short-time Fourier transform. SIAM J. Appl. Math. 72(5), 1449-1473 (2012) 
4. de Branges, L.: Hilbert spaces of entire functions. Prentice-Hall Inc, Englewood Cliffs (1968)

5. Dym, H.: An introduction to de Branges spaces of entire functions with applications to differential equations of the Sturm-Liouville type. Adv. Math. 5, 395-471 (1970/1971)

6. Dym, H., McKean, H.P.: Gaussian processes, function theory, and the inverse spectral problem (Probability and Mathematical Statistics), vol. 31. Academic Press, New York (1976)

7. García, A.G., Hernández-Medina, M.A., Szafraniec, F.H.: Analytic Kramer kernels, Lagrange-type interpolation series and de Branges spaces. Complex Var. Elliptic Equ. 58(1), 79-97 (2013)

8. Jorgensen, P., Tian, F.: Nonuniform sampling, reproducing kernels, and the associated Hilbert spaces. Sampl. Theory Signal Image Process. 15, 37-72 (2016)

9. Kadets, M.I.: The exact value of the Paley-Wiener constant. Dokl. Akad. Nauk SSSR 155, 1253-1254 (1964)

10. Kaltenbäck, M., Woracek, H.: Pontryagin spaces of entire functions I. Integral Equ. Oper. Theory 33(1), 34-97 (1999)

11. Kostenko, A., Sakhnovich, A., Teschl, G.: Inverse eigenvalue problems for perturbed spherical Schrödinger operators. Inverse Probl. 26(10), 105013 (2010)

12. Kozachenko, Y., Olenko, A.: Aliasing-truncation errors in sampling approximations of sub-Gaussian signals. IEEE Trans. Inform. Theory 62(10), 5831-5838 (2016)

13. Levitan, B.M., Sargsjan, I.S.: Sturm-Liouville and Diracoperators, Volume 59 of Mathematics and its Applications(Soviet Series). Kluwer Academic Publishers Group, Dordrecht (Translated from the Russian) (1991)

14. Papoulis, A.: Error analysis in sampling theory. Proc. IEEE 54(7), 947-955 (1966)

15. Partington, J.R.: Interpolation, Identification, and Sampling, Volume 17 of London Mathematical Society Monographs. NewSeries. Oxford University Press, New York (1997)

16. Pöschel, J., Trubowitz, E.: Inverse Spectral Theory, Volume 130 of Pure and Applied Mathematics. Academic, Boston (1987)

17. Remling, C.: Schrödinger operators and de Branges spaces. J. Funct. Anal. 196(2), 323-394 (2002)

18. Silva, L.O., Toloza, J.H.: Applications of Krein's theory of regular symmetric operators to sampling theory. J. Phys. A 40(31), 9413-9426 (2007)

19. Silva, L.O., Toloza, J.H.: The class of $n$-entire operators. J. Phys. A 46(2), 025202 (2013)

20. Silva, L.O., Toloza, J.H.: A class of $n$-entire Schrödinger operators. Complex Anal. Oper. Theory 8(8), 1581-1599 (2014)

21. Silva, L.O., Toloza, J.H.: De Branges spaces and KreĬn's theory of entire operators. In: Operator Theory. With 51 Figures and 2 Tables in volumes 2, pp. 549-580. Springer, Basel (2015)

22. Teschl, G.: Mathematical Methods in Quantum Mechanics, Volume 99 of Graduate Studies in Mathematics (With Applications to Schrödinger Operators). American Mathematical Society, Providence (2009)

23. Zayed, A.I.: Advances in Shannon's Sampling Theory. CRC Press, Boca Raton (1993) 